\title{
Misturas BR/SBR: Propriedades Mecânicas em Função do Modo de Preparo
}

\author{
Adriana F. de Alcantara, Regina Célia R. Nunes, Leila L. Y. Visconte \\ IMA, UFRJ
}

Resumo: Misturas com borracha são freqüentemente usadas para se conseguir o balanço das propriedades desejadas e também, em alguns casos, a redução de custos. As misturas elastoméricas são normalmente sistemas multifásicos e a distribuição dos aditivos entre as fases não é necessariamente uniforme. Neste trabalho, o polibutadieno (BR) foi misturado ao elastômero de butadieno-estireno (SBR) na proporção 1:1 em peso. As composições foram preparadas utilizando um misturador de rolos, segundo a norma ASTM D3182, sendo que a incorporação dos aditivos foi realizada de quatro formas diferentes. Após a avaliação das propriedades reométricas e a vulcanização, foram estudadas as propriedades mecânicas para cada composição, avaliadas de acordo com as normas ASTM específicas para cada ensaio. Os resultados mostram que os diferentes modos de preparo de uma mesma formulação podem provocar mudança significativa nas propriedades mecânicas de misturas envolvendo essas duas borrachas. No caso das misturas BR/SBR estudadas, as propriedades apresentaram valores intermediários aos das borrachas isoladas.

Palavras-chave: Misturas de elastômeros, propriedades mecânicas, $B R, S B R$.

\section{BR/SBR Blends: Mechanical Properties as a Function of the Preparation Mode}

Abstract: Rubber blends are frequently prepared to give a balance of the desired properties and, in some cases, reduction of cost as well. Elastomeric mixtures are usually multiphase systems and the dispersion of the additives may not be uniform throughout the phases. In this work, polybutadiene rubber (BR) was mixed with polybutadiene-styrene rubber (SBR) in a 50:50 w/w ratio. The compositions were prepared in a two-roll mill, according to ASTM D3182, and the incorporation of the ingredients was carried out according to four different modes. After the determination of the rheometric properties and the vulcanization of the compounds, mechanical properties were evaluated according to specific ASTM procedures. The results show that the mechanical properties may be altered by changing the procedure to prepare a given formulation. In the case of BR/SBR mixtures, the properties were found to be intermediate to the values presented by the compositions with the separate rubbers.

Keywords: Elastomer blends, mechanical properties, BR, $S B R$.

\section{Introdução}

Na geração de novos materiais poliméricos com aplicação comercial e utilidade prática, é muito comum a utilização de misturas de dois ou mais elastômeros em uma composição, uma vez que este procedimento é mais acessível e menos dispendioso do que o desenvolvimento de novos polímeros ${ }^{[1]}$.

A verdadeira razão para que se faça a mistura de dois elastômeros é combinar duas ou mais características desejáveis, exibidas pelos vulcanizados dos elastômeros individuais, em um único material. Embora isto seja aparentemente fácil de ser obtido para algumas misturas, freqüentemente o resultado é menos satisfatório do que o esperado e pode ser inferior, em alguns aspectos, às propriedades dadas pelos elastômeros individuais. O processo de otimizar um número apreciável de variáveis, de modo a se obter o melhor ajuste de uma faixa de propriedades para uma aplicação em particular, é uma prática normal e faz parte do desenvolvimento de uma formulação para uma borracha vulcanizada ${ }^{[2]}$.

Misturas com borracha são freqüentemente usadas para: melhorar as suas propriedades, melhorar o seu processamento e/ou reduzir custos. As propriedades físicas das misturas não são determinadas somente pelas propriedades dos componentes individuais, mas também pela sua estrutura física ${ }^{[3]}$. Assim, para que as propriedades sejam aquelas esperadas, deve-se levar em consideração dois fatores importantes: o sistema de vulcanização e a compatibilização ${ }^{[3]}$.

Neste trabalho foram empregadas as borrachas BR e SBR que possuem características muito parecidas. Esses dois elastômeros foram misturados na proporção de 1:1 em peso e a mistura destes dois elastômeros e os demais ingredientes da formulação foi realizada de quatro maneiras distintas. A partir das composições obtidas foram analisadas as propriedades mecânicas em função do método de mistura.

Autor para correspondência: Leila L. Y. Visconte, Instituto de Macromoléculas Professora Eloisa Mano, UFRJ, Caixa Postal 68.525, CEP:21.945-970, Rio de Janeiro, RJ, Brasil.E-mail: lyv@ima.ufrj.br 


\section{Experimental}

No presente trabalho foram utilizadas as borrachas de estireno-butadieno (SBR), tipo 1502, e a borracha de polibutadieno (BR), tipo BR-40, com alto teor de unidades cis, ambas produzidas pela Petroflex S.A. Indústria e Comércio. Todos os materiais foram usados como recebidos.

A seguinte formulação foi utilizada na preparação das misturas (em phr): elastômeros - BR (50) e SBR (50); ativadores - óxido de zinco $(3,0)$ e ácido esteárico $(2,0)$; antioxidante - octamine, 4,4'-dioctil-difenilamina $(1,5)$; agente de vulcanização - enxofre $(1,8)$; aceleradores TBBS, t-butil-2-benzotiazol sulfenamida $(1,2)$ e DPG, difenil-guanidina $(0,10)$; óleo aromático $(3,75)$. As misturas foram preparadas em misturador de rolos Berstoff, a $50{ }^{\circ} \mathrm{C}$, empregando-se quatro modos diferentes para a incorporação dos aditivos:

M1: os aditivos, exceto os aceleradores, foram misturados inicialmente na borracha SBR e, após a homogeneização, o BR foi adicionado. Quando a mistura tornou-se homogênea, acrescentaram-se os aceleradores.

M2: os aditivos, exceto os aceleradores, foram misturados inicialmente na borracha $\mathrm{BR}$ e, após a homogeneização, $\mathrm{o}$ SBR foi adicionado. Quando a mistura tornou-se homogênea, acrescentaram-se os aceleradores.

M3: as borrachas BR e SBR foram previamente misturadas e após a homogeneização, os aditivos foram acrescentados.

M4: a cada uma das borrachas foi adicionada metade da quantidade de cada aditivo da formulação, exceto os aceleradores. As duas composições foram então misturadas para homogeneização, sendo por último adicionados os aceleradores.

Vinte e quatro horas após a preparação no misturador de rolos, as diferentes composições foram analisadas para a determinação dos parâmetros reométricos, em reômetro de disco oscilatório (Reômetro Monsanto, modelo 100S), operando com arco de $3^{\circ}$ e temperatura de $160^{\circ} \mathrm{C}$.

Os ensaios de tração e rasgamento foram realizados em dinamômetro Instron, segundo as normas ASTM D $412^{[4]}$ e D $624^{[5]}$, respectivamente, à temperatura ambiente, com velocidade de deformação de $500 \mathrm{~mm} / \mathrm{min}$. Para os ensaios de tração e rasgamento foram utilizados corpos-de-prova modelo $\mathrm{C}$. Os espécimes foram extraídos de placas vulcanizadas a partir do tempo ótimo de cura $\left(\mathrm{t}_{90}\right)$, obtido através dos parâmetros reométricos.

A dureza das amostras foi determinada em durômetro Shore A, de acordo com a norma ASTM D2240 ${ }^{[6]}$, utilizada para a determinação da dureza de amostras flexíveis.

Para a deformação permanente à compressão, a metodologia empregada seguiu a norma ASTM D395 $5^{[7]}$, utilizando-se as seguintes condições: $70^{\circ} \mathrm{C}$, em estufa, por um período de 22 horas.

As medidas de resiliência foram determinadas em um Resiliômetro Bashore, de acordo com a norma ASTM D2632 $2^{[8]}$. Como a escala do resiliômetro é dividida em 100 partes, a resiliência foi expressa em percentagem.

\section{Resultados e Discussão}

As propriedades mecânicas são apresentadas nas Figuras 1-6.

A Figura 1 mostra que o vulcanizado contendo BR apresenta maior dureza que aquele com SBR. A figura mostra também que a composição obtida pelo método M1 apresenta maior dureza que as demais, e supera os valores apresentados pelas borrachas isoladas. As demais misturas não foram influenciadas pelo método de preparo, apresentando dureza semelhante à composição com BR puro.

Os valores para a deformação permanente à compressão são apresentadas na Figura 2, onde se observa o maior valor fornecido pelo vulcanizado de SBR em relação ao BR, para esta propriedade. Os valores de deformação apresentados pelas misturas BR/SBR são intermediários aos obtidos pelas borrachas isoladas. Aparentemente, o modo M4, pelo qual metade da quantidade de cada ingrediente é adicionado a cada uma das borrachas antes da mistura, é a maneira mais eficaz de se preservar as boas propriedades elásticas da BR. $\mathrm{O}$ fato parece sugerir que essas propriedades são fortemente dependentes de uma boa distribuição dos aditivos em cada uma das fases.

A Figura 3 mostra a superioridade do SBR em comparação com o BR, com relação a resistência à tração. Já para as misturas dos dois elastômeros obtidas pelos diferentes métodos, observa-se que esta propriedade não varia significativamente e que todos os valores apresentados são intermediários

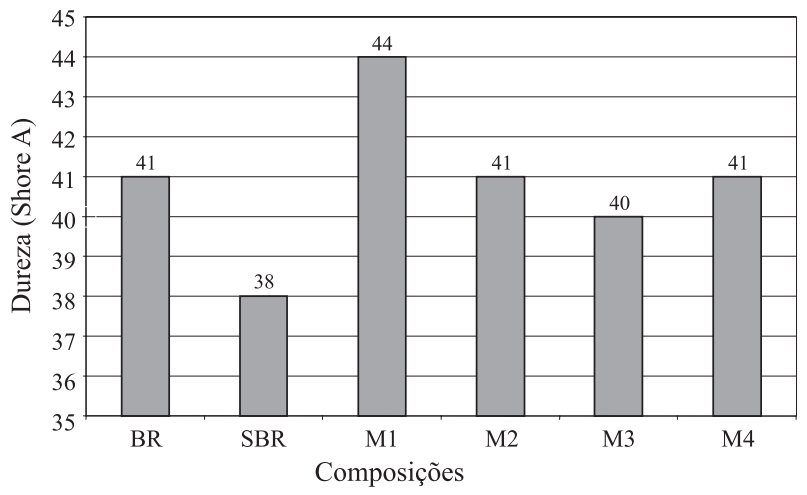

Figura 1. Dureza Shore de misturas BR/SBR

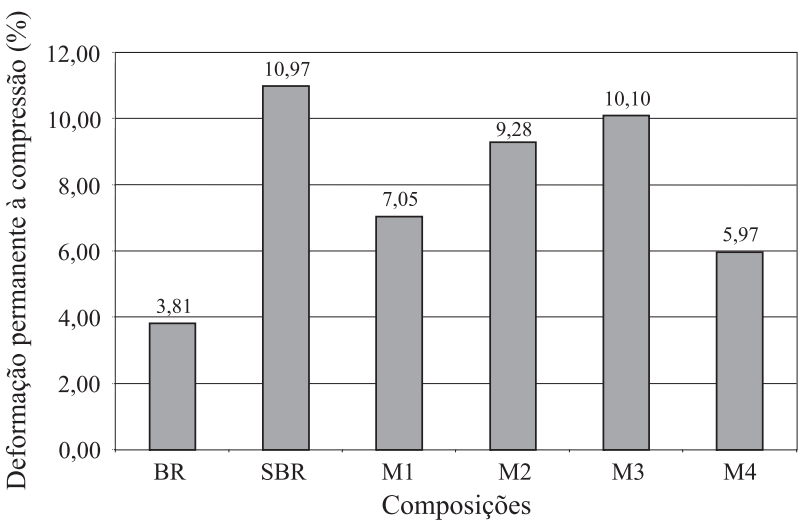

Figura 2. Deformação permanente à compressão das misturas BR/SBR 
aos das borrachas isoladas. Ainda assim, o melhor resultado é dado por M2, em que os aditivos são adicionados ao BR, estimulando, dessa forma, a vulcanização desta fase.

Os resultados para o alongamento na ruptura das composições podem ser vistos na Figura 4, onde se verifica que essa propriedade não é afetada por mudanças na ordem de adição dos ingredientes da formulação, ficando todos os valores intermediários aos apresentados pelas gomas puras.

A Figura 5 mostra a superioridade da borracha SBR em relação à BR quanto ao rasgamento. Verifica-se que os valores encontrados para as misturas de ambas as borrachas, embora intermediários aos das borrachas isoladas, estão mais próximos ao resultado apresentado pelo SBR. Percebe-se que quando a adição total (M2) ou mesmo parcial (M4) de todos os ingredientes da formulação à fase BR é feita, esta propriedade fica ligeiramente prejudicada, ao contrário do que acontece quando é a fase SBR que recebe os ingredientes. Isso significa que para se obter uma boa resistência ao rasgamento, a fase SBR deve apresentar alto grau de vulcanização.

Levando-se em conta os quatro métodos de mistura estudados, a ordem de incorporação dos ingredientes não altera significativamente a resiliência, como mostra a Figura 6, mas verifica-se que ao se misturar as borrachas BR e SBR, as composições tendem a apresentar valores de resiliência igual ou superior à apresentada pela composição com $\mathrm{BR}$ isolada. Isso mostra que a alta resiliência da BR é mantida, mesmo em presença da borracha SBR.

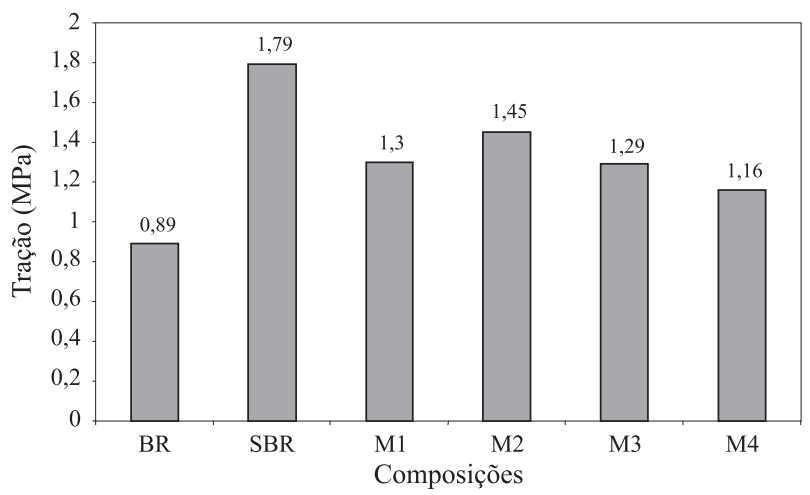

Figura 3. Tração na ruptura de composições BR/SBR

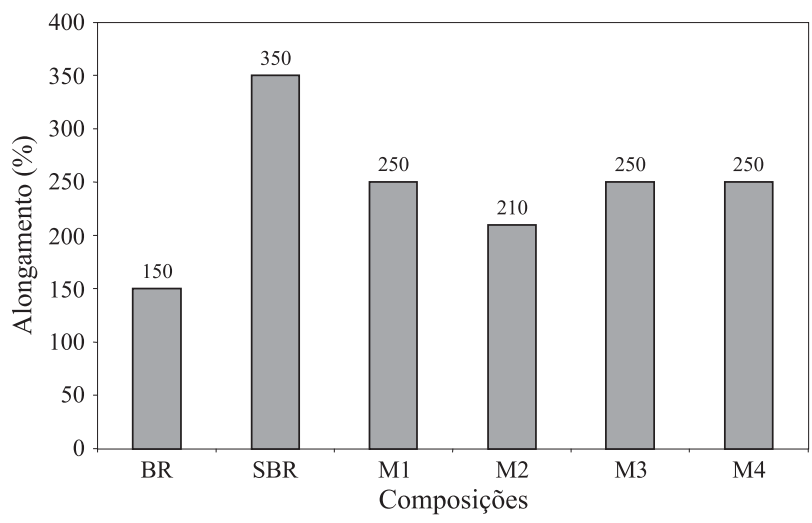

Figura 4. Alongamento na ruptura de composições BR/SBR

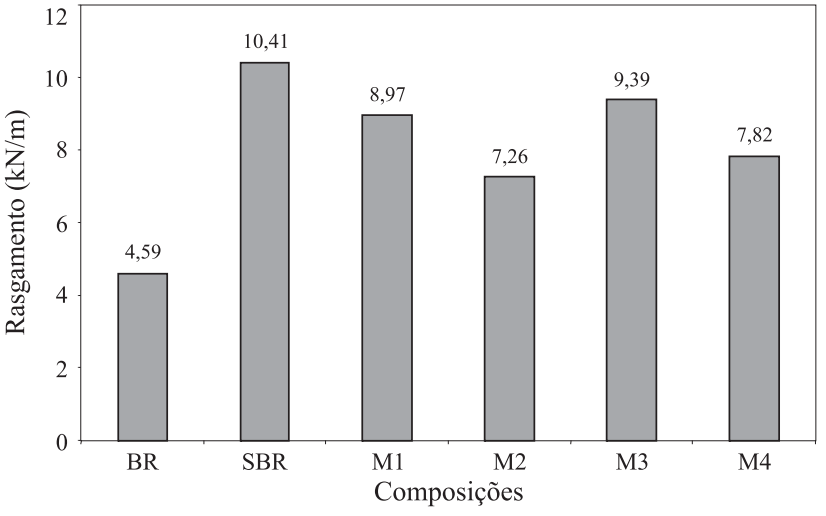

Figura 5. Resistência ao rasgamento de composições BR/SBR

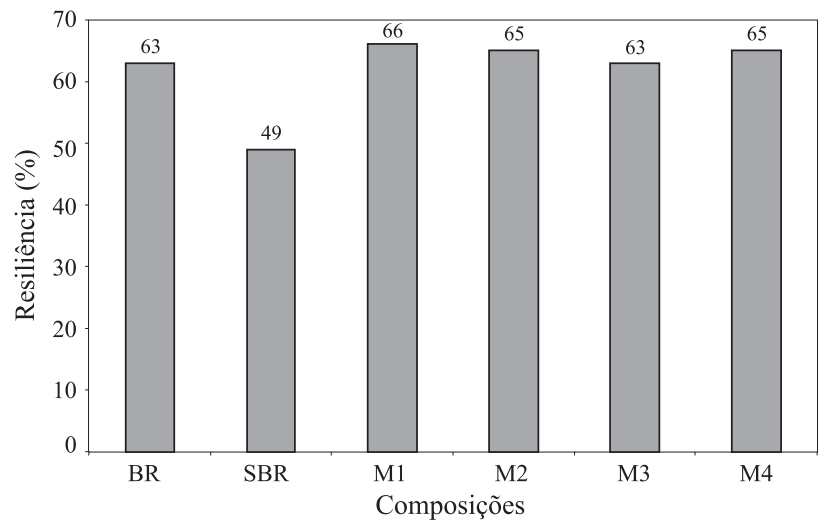

Figura 6. Resiliência de misturas BR/SBR

\section{Conclusões}

A maneira como os ingredientes de uma formulação são adicionados a uma mistura de borrachas pode ter grande influência sobre suas propriedades mecânicas. Quando BR e SBR são misturadas de quatro maneiras diferentes, na proporção de 1:1 em peso, as propriedades são em geral muito parecidas, intermediárias às apresentadas pelas borrachas isoladas. Uma exceção notável diz respeito à resiliência, cujos valores encontrados são iguais ou superiores ao da composição com BR apenas.

\section{Agradecimentos}

Os autores agradecem à Petroflex S. A. Comércio e Indústria pela doação das borrachas e ao CNPq pelo apoio financeiro.

\section{Referências Bibliográficas}

1. Roe, R. J. - "Glass Transition”, in Encyclopedia of Polymer Science and Engineering, vol. 14, Mark, H. F.; Bikales, N. M.; Overberger, C. G. \& Menges, G. (ed.), John Wiley \& Sons, New York (1989)

2. Tinker, A. J. - Rubb. Chem. Technol., 68, p.461 (1995).

3. Huson, M. G.; McGill, W. J.; Swart, P. J. - J. Polym. Sci.: Polym. Lett. Ed., 22, p.143 (1984). 
4. American Society for Testing and Materials, ASTM D412-87, "Standard test method for rubber properties in tension"; vol. 9.01, Philadelphia, (1986).

5. American Society for Testing and Materials, ASTM D624-86, "Standard test method for rubber property Tear resistence"; vol. 9.01, Philadelphia, (1986).

6. American Society for Testing and Materials, ASTM D2240-86, "Standard test method for rubber property - Durometer hardness"; vol. 9.01, Philadelphia, (1986).
7. American Society for Testing and Materials, ASTM D39585 , "Standard test method for rubber property Compression set"; vol. 9.01, Philadelphia, (1986).

8. American Society for Testing and Materials, ASTM D263288, "Standard test method for rubber property Resilience by vertical rebound"; vol. 9.01, Philadelphia, (1986).

Enviado: 23/06/04

Aprovado: 20/08/04 